\title{
Mathematical Morphological Distributive Concepts over Unions and Intersections
}

\author{
Joseph Ackora-Prah*, Robert K. Acquah, Yao Elikem Ayekple \\ Department of Mathematics, Kwame Nkrumah University of Science and Technology, Kumasi, Ghana \\ Email: ${ }^{* j a a c k o r a-p r a h . c o s @ k n u s t . e d u . g h ~}$
}

How to cite this paper: Ackora-Prah, J., Acquah, R.K. and Ayekple, Y.E. (2016) Mathematical Morphological Distributive Concepts over Unions and Intersections. Advances in Pure Mathematics, 6, 633-637. http://dx.doi.org/10.4236/apm.2016.610052

Received: April 6, 2016

Accepted: September 10, 2016

Published: September 13, 2016

Copyright $\odot 2016$ by authors and Scientific Research Publishing Inc. This work is licensed under the Creative Commons Attribution International License (CC BY 4.0). http://creativecommons.org/licenses/by/4.0/

\begin{abstract}
Mathematical Morphological concepts outline techniques for analysing and processing geometric structures based on set theory. In this paper, we present proofs of our theorems on morphological distributive properties over Unions and Intersections with respect to Dilation and Erosion. These results provide new realizations of Dilation, Erosion and conclude that they are distributive over Unions but non-distributive over Intersections.
\end{abstract}

\section{Keywords}

Mathematical Morphology, Dilation, Distributive

\section{Introduction}

Mathematical Morphology is a tool for the extraction of components of images used to describe and represent skeletons, boundaries etc., which involves techniques like morphological thinning, pruning and filtering. Morphological concepts date back to works done by Matheron and Minkowski who used binary mathematical morphology on integral geometry [1], [2]. Matheron and Serra also used the techniques in texture and image analysis [1], [3]. In our recent paper titled "Revised Mathematical Morphological Concepts" [4], we outlined in details some mathematical morphological operators and their algebraic structures when they are linked with unions and intersections. We showed that the partitioning of structural elements before morphological operations is possible. In this paper, we present results on the distributive properties of Dilation and Erosion over unions and intersections. This paper is a continuation of the revised mathematical morphological concept [4] and hence most of the concepts that were developed and discussed in it will be used here without explaining. Therefore, we urge that you read it before going through this paper. 


\section{Preliminaries}

\subsection{Definitions}

The following definitions are important for our purpose.

Definition 1 (Dilation) Let the image set $X$ and the structuring element $B$ be subsets of the discrete space $Z^{2}: X \subset Z^{2}, B \subset Z^{2}$. The dilation of $X$ by $B$ is defined as $X \oplus B=\left\{c=x+b \in Z^{2}: x \in X ; b \in B\right\}$; or the Dilation of a binary image $A$ by structure element $B$, is $A \oplus B=\{a+b \mid$ for $a \in A$ and $b \in B\}$.

The dilation transform generally causes image objects to grow in size. From the definitions above, dilation is equivalent to a union of translates of the original image with respect to the structure element, that is, $A \oplus B=\bigcup_{b \in B} A_{b}$.

Definition 2 (Erosion) Let the image set $X$ and the structuring element $B$ be subsets of the discrete space $Z^{2}: X \subset Z^{2}, B \subset Z^{2}$. The erosion of $X$ by $B$ is defined as $X \ominus B=\left\{c \in Z^{2}: \forall b \in B, \exists x \in X\right.$ where $\left.c=x-b\right\}$; or the Erosion of a binary image $A$ by structure element $B$, is $A \ominus B=\{p \mid p+b \in A \forall b \in B\}$.

Similarly erosion transform allows image objects to shrink in size, that is, $A \ominus B=\bigcap_{b \in B} A_{-b}$.

\subsection{Algebraic Properties of Dilation and Erosion}

We note that Dilation is commutative and associative, that is, $A \oplus B=B \oplus A$ and $(A \oplus B) \oplus C=A \oplus(B \oplus C)$, where as Erosion is non-commutative and non-associative, that is, $A \ominus B \neq B \ominus A$ and $(A \ominus B) \ominus C \neq A \ominus(B \ominus C)$ respectively.

Furthermore, Dilation and Erosion are both translation invariant, that is, if $x$ is a vector belonging to $A$ and $B(x \in A, x \in B)$, then $A \oplus B_{x}=A_{x} \oplus B=(A \oplus B)_{x}$ and $A \ominus B_{x}=A_{x} \ominus B=(A \ominus B)_{x}$. Also both Dilation and Erosion are increasing in $A$, that is, if an image set $A_{1}$ is a subset of $A_{2}\left(A_{1} \subset A_{2}\right)$, then $A_{1} \oplus B \subset A_{2} \oplus B$ and $A_{1} \ominus B \subset A_{2} \ominus B$. However, Erosion is decreasing in $B$, that is, if a structuring element $B_{1}$ is a subset of $B_{2}\left(B_{1} \subset B_{2}\right)$, then $A \ominus B_{1} \supset A \ominus B_{2}$. Dilation and Erosion transforms are duals of each other, that is, $(A \oplus B)^{c}=A^{c} \ominus \breve{B}$ and $(A \ominus B)^{c}=A^{c} \oplus \breve{B}$. Dilation and Erosion are also not the inverse of each other, that is, $(A \oplus B) \ominus B \neq A$ and $(A \ominus B) \oplus B \neq A$. Both the dilation and erosion transforms have an identity set, $I$, such that $A \oplus I=A$ and $A \ominus I=A$. Dilation transform has an empty set, that is, $\varnothing$ such that $A \oplus \varnothing=\varnothing$.

\section{Results}

We present results of the distribution of morphological operators over set unions and intersections of two different sets and their extensions. The theorems and their proofs below will facilitate the understanding of the various results.

The Distribution of Morphological Operators over Set Union and Intersection

Theorem 1 (The distribution of Dilation over union with $\mathbf{n}$ distinct structural elements) 
If $B=B_{1} \cup B_{2} \cup B_{3} \cup \cdots \cup B_{n}$ for $n \geq 2$

Then $A \oplus B=\left(A \oplus B_{1}\right) \cup\left(A \oplus B_{2}\right) \cup\left(A \oplus B_{3}\right) \cup \cdots \cup\left(A \oplus B_{n}\right)$

\section{Proof:}

If $B=B_{1} \cup B_{2}$

$$
\begin{aligned}
\left(A \oplus B_{1}\right) \cup\left(A \oplus B_{2}\right) & =\bigcup_{b_{1} \in B_{1}} A_{b_{1}} \cup \bigcup_{b_{2} \in B_{2}} A_{b_{2}} \\
& =\bigcup_{b \in B_{1} \cup B_{2}}\left\{A_{b} \cap A_{b_{1}}\right\} \cup \bigcup_{b \in B_{1} \cup B_{2}}\left\{A_{b} \cap A_{b_{2}}\right\} \\
& =\bigcup_{b \in B_{1} \cup B_{2}}\left\{A_{b} \cap A_{b_{1}} \cup A_{b} \cap A_{b_{2}}\right\} \\
& =\bigcup_{b \in B_{1} \cup B_{2}} A_{b}=A \oplus\left(B_{1} \cup B_{2}\right)=A \oplus B
\end{aligned}
$$

This implies $A \oplus B=\left(A \oplus B_{1}\right) \cup\left(A \oplus B_{2}\right)$

Let assume that if $B=B_{1} \cup B_{2} \cup B_{3} \cup \cdots \cup B_{k}$ for $k \geq 2$

Then $A \oplus B=\left(A \oplus B_{1}\right) \cup\left(A \oplus B_{2}\right) \cup\left(A \oplus B_{3}\right) \cup \cdots \cup\left(A \oplus B_{k}\right)$

Now we show that if $B=B_{1} \cup B_{2} \cup B_{3} \cup \cdots \cup B_{k} \cup B_{k+1}$

Then

$$
\begin{aligned}
A \oplus B & =A \oplus\left(B_{1} \cup B_{2} \cup B_{3} \cup \cdots \cup B_{k} \cup B_{k+1}\right) \\
& =A \oplus\left[\left(B_{1} \cup B_{2} \cup B_{3} \cup \cdots \cup B_{k}\right) \cup B_{k+1}\right] \\
& =\left[A \oplus\left(B_{1} \cup B_{2} \cup B_{3} \cup \cdots \cup B_{k}\right)\right] \cup\left(A \oplus B_{k+1}\right) \\
& =\left(A \oplus B_{1}\right) \cup\left(A \oplus B_{2}\right) \cup\left(A \oplus B_{3}\right) \cup \cdots \cup\left(A \oplus B_{k}\right) \cup\left(A \oplus B_{k+1}\right)
\end{aligned}
$$

Theorem 2 (The distribution of Erosion over union with $\mathrm{n}$ distinct structural elements)

If $B=B_{1} \cup B_{2} \cup B_{3} \cup \cdots \cup B_{n}$ for $n \geq 2$

Then $A \ominus B=\left(A \ominus B_{1}\right) \cup\left(A \ominus B_{2}\right) \cup\left(A \ominus B_{3}\right) \cup \cdots \cup\left(A \ominus B_{n}\right)$

\section{Proof:}

If $B=B_{1} \cup B_{2}$

$$
\begin{aligned}
\left(A \ominus B_{1}\right) \cup\left(A \ominus B_{2}\right) & =\bigcap_{b_{1} \in B_{1}} A_{-b_{1}} \cup \bigcap_{b_{2} \in B_{2}} A_{-b_{2}} \\
& =\bigcap_{b \in B_{1} \cup B_{2}}\left\{A_{-b} \cap A_{-b_{1}}\right\} \cup \bigcap_{b \in B_{1} \cup B_{2}}\left\{A_{-b} \cap A_{-b_{2}}\right\} \\
& =\bigcap_{b \in B_{1} \cup B_{2}}\left\{A_{-b} \cap A_{-b_{1}} \cup A_{-b} \cap A_{-b_{2}}\right\} \\
& =\bigcap_{b \in B_{1} \cup B_{2}} A_{-b}=A \ominus\left(B_{1} \cup B_{2}\right)=A \ominus B
\end{aligned}
$$

This implies $A \ominus B=\left(A \ominus B_{1}\right) \cup\left(A \ominus B_{2}\right)$

Let assume that if $B=B_{1} \cup B_{2} \cup B_{3} \cup \cdots \cup B_{k}$ for $k \geq 2$

Then $A \ominus B=\left(A \ominus B_{1}\right) \cup\left(A \ominus B_{2}\right) \cup\left(A \ominus B_{3}\right) \cup \cdots \cup\left(A \ominus B_{k}\right)$

Now we show that if $B=B_{1} \cup B_{2} \cup B_{3} \cup \cdots \cup B_{k} \cup B_{k+1}$

Then

$$
\begin{aligned}
A \ominus B & =A \ominus\left(B_{1} \cup B_{2} \cup B_{3} \cup \cdots \cup B_{k} \cup B_{k+1}\right) \\
& =A \ominus\left[\left(B_{1} \cup B_{2} \cup B_{3} \cup \cdots \cup B_{k}\right) \cup B_{k+1}\right] \\
& =\left[A \ominus\left(B_{1} \cup B_{2} \cup B_{3} \cup \cdots \cup B_{k}\right)\right] \cup\left(A \ominus B_{k+1}\right) \\
& =\left(A \ominus B_{1}\right) \cup\left(A \ominus B_{2}\right) \cup\left(A \ominus B_{3}\right) \cup \cdots \cup\left(A \ominus B_{k}\right) \cup\left(A \ominus B_{k+1}\right)
\end{aligned}
$$

The dilation of a set of two different structural elements and taking the union is the 
same as taking the union of the structural element and dilating with the set. This shows that morphological dilation distributes over set unions. It also leads to the fact that; if any structural element can be partitioned into $n$ distinct parts then the union of each of the partitions dilation with the set is the same as the set's dilation with the structural element. We note also that provided any structural element can be partitioned into $n$ distinct parts, then the union of each of the partition's erosion with the set is equal to the set's erosion with the structural element.

Theorem 3 (Non-distribution of Erosion over intersection)

If $B=B_{1} \cup B_{2} \cup B_{3} \cup \cdots \cup B_{n}$ for $n \geq 2$

Then $A \ominus B=\left(A \ominus B_{1}\right) \cap\left(A \ominus B_{2}\right) \cap\left(A \ominus B_{3}\right) \cap \cdots \cap\left(A \ominus B_{n}\right)$

Proof:

If $B=B_{1} \cup B_{2}$

$$
\begin{aligned}
{\left[\left(A \ominus B_{1}\right) \cap\left(A \ominus B_{2}\right)\right]^{c} } & =\left(A \ominus B_{1}\right)^{c} \cup\left(A \ominus B_{2}\right)^{c} \\
& =\left(A^{c} \oplus \breve{B}_{1}\right) \cup\left(A^{c} \oplus \breve{B}_{2}\right) \\
& =A^{c} \oplus\left(\breve{B}_{1} \cup \breve{B}_{2}\right) \\
\Rightarrow\left(A \ominus B_{1}\right) \cap\left(A \ominus B_{2}\right)= & {\left[A^{c} \oplus\left(\breve{B}_{1} \cup \breve{B}_{2}\right)\right]^{c} } \\
& =A \ominus\left(B_{1} \cup B_{2}\right)=A \ominus B
\end{aligned}
$$

Then

This implies $A \ominus B=\left(A \ominus B_{1}\right) \cap\left(A \ominus B_{2}\right)$

Let assume that if $B=B_{1} \cup B_{2} \cup B_{3} \cup \cdots \cup B_{k}$ for $k \geq 2$

Then $A \ominus B=\left(A \ominus B_{1}\right) \cap\left(A \ominus B_{2}\right) \cap\left(A \ominus B_{3}\right) \cap \cdots \cap\left(A \ominus B_{k}\right)$

Now we show that if $B=B_{1} \cup B_{2} \cup B_{3} \cup \cdots \cup B_{k} \cup B_{k+1}$

Then

$$
\begin{aligned}
A \ominus B & =A \ominus\left(B_{1} \cup B_{2} \cup B_{3} \cup \cdots \cup B_{k} \cup B_{k+1}\right) \\
& =A \ominus\left[\left(B_{1} \cup B_{2} \cup B_{3} \cup \cdots \cup B_{k}\right) \cup B_{k+1}\right] \\
& =\left[A \ominus\left(B_{1} \cup B_{2} \cup B_{3} \cup \cdots \cup B_{k}\right)\right] \cap\left(A \ominus B_{k+1}\right) \\
& =\left(A \ominus B_{1}\right) \cap\left(A \ominus B_{2}\right) \cap\left(A \ominus B_{3}\right) \cap \cdots \cap\left(A \ominus B_{k}\right) \cap\left(A \ominus B_{k+1}\right)
\end{aligned}
$$

\section{Theorem 4 (Non-distribution of Dilation over intersection)}

If $B=B_{1} \cup B_{2} \cup B_{3} \cup \cdots \cup B_{n}$ for $n \geq 2$

Then $A \oplus B=\left(A \oplus B_{1}\right) \cap\left(A \oplus B_{2}\right) \cap\left(A \oplus B_{3}\right) \cap \cdots \cap\left(A \oplus B_{n}\right)$

\section{Proof:}

If $B=B_{1} \cup B_{2}$

$$
\begin{aligned}
{\left[\left(A \oplus B_{1}\right) \cap\left(A \oplus B_{2}\right)\right]^{c} } & =\left(A \oplus B_{1}\right)^{c} \cup\left(A \oplus B_{2}\right)^{c} \\
& =\left(A^{c} \ominus \breve{B}_{1}\right) \cup\left(A^{c} \ominus \breve{B}_{2}\right) \\
& =A^{c} \ominus\left(\breve{B}_{1} \cup \breve{B}_{2}\right) \\
\Rightarrow\left(A \oplus B_{1}\right) \cap\left(A \oplus B_{2}\right)= & {\left[A^{c} \ominus\left(\breve{B}_{1} \cup \breve{B}_{2}\right)\right]^{c} } \\
& =A \oplus\left(B_{1} \cup B_{2}\right)=A \oplus B
\end{aligned}
$$

Then

This implies $A \oplus B=\left(A \oplus B_{1}\right) \cap\left(A \oplus B_{2}\right)$

Let assume that if $B=B_{1} \cup B_{2} \cup B_{3} \cup \cdots \cup B_{k}$ for $k \geq 2$

Then, $A \oplus B=\left(A \oplus B_{1}\right) \cap\left(A \oplus B_{2}\right) \cap\left(A \oplus B_{3}\right) \cap \cdots \cap\left(A \oplus B_{k}\right)$ 
Now, we show that if $B=B_{1} \cup B_{2} \cup B_{3} \cup \cdots \cup B_{k} \cup B_{k+1}$

$$
\begin{aligned}
A \oplus B & =A \oplus\left(B_{1} \cup B_{2} \cup B_{3} \cup \cdots \cup B_{k} \cup B_{k+1}\right) \\
& =A \oplus\left[\left(B_{1} \cup B_{2} \cup B_{3} \cup \cdots \cup B_{k}\right) \cup B_{k+1}\right] \\
& =\left[A \oplus\left(B_{1} \cup B_{2} \cup B_{3} \cup \cdots \cup B_{k}\right)\right] \cap\left(A \oplus B_{k+1}\right) \\
& =\left(A \oplus B_{1}\right) \cap\left(A \oplus B_{2}\right) \cap\left(A \oplus B_{3}\right) \cap \cdots \cap\left(A \oplus B_{k}\right) \cap\left(A \oplus B_{k+1}\right)
\end{aligned}
$$

Then,

The intersection of the erosion of a set with structural elements is equivalent to the union of the structural elements on the erosion of the set. We note that since we are supposed to take the union instead of the intersection, it shows that morphological erosion is non-distributed over set intersection. Similar arguments hold for dilation which leads to the non-distributive property of dilation over intersection.

\section{Conclusion}

We have presented theorems and their proofs on morphological distribution properties over unions and intersections. Our results show that Dilation and Erosion are distributive over unions but non-distributive over intersections. In addition, our theorems facilitate the partitioning of structural elements in order to implement morphological operations.

\section{References}

[1] Matheron, G. (1975) Random Sets and Integral Geometry. Wiley, New York.

[2] Minkowski, H. (1903) Vorlumen und Oberflache. Mathematische Annalen, 57, 447-495. http://dx.doi.org/10.1007/BF01445180

[3] Serra, J.C. (1982) Image Analysis and Mathematical Morphology. Academic Press, New York.

[4] Ackora-Prah, J., Ayekple, E., Acquah, R., Andam, P., Sakyi, E. and Gyamfi, D. (2015) Revised Mathematical Morphological Concepts. Advances in Pure Mathematics, 5, 155-161. http://dx.doi.org/10.4236/apm.2015.54019

\section{Scientific Research Publishing}

\section{Submit or recommend next manuscript to SCIRP and we will provide best service for you:}

Accepting pre-submission inquiries through Email, Facebook, LinkedIn, Twitter, etc. A wide selection of journals (inclusive of 9 subjects, more than 200 journals)

Providing 24-hour high-quality service

User-friendly online submission system

Fair and swift peer-review system

Efficient typesetting and proofreading procedure

Display of the result of downloads and visits, as well as the number of cited articles

Maximum dissemination of your research work

Submit your manuscript at: http://papersubmission.scirp.org/ 